\title{
Les postes hydrauliques de vallées au Groupe régional de production hydraulique "Alpes »
}

\author{
Pascal Verrier \\ EDF-G.R.P.H. - «Alpes »
}

Présentation des postes hydrauliques de vallées

Afin d'améliorer la qualité du service rendu aux Centres des mouvements d'énergie et de faciliter l'exploitation des aménagements (mise en place d'un service de quart centralisé), le Service de la production hydraulique a décidé de réaliser une nouvelle génération de postes de commande hydraulique baptisés postes hydrauliques de vallées ou P.H.V.

Un P.H.V. est donc un système de commande informatique hiérarchisé, constitué d'une part, d'un calculateur central appelé poste de commande, et d'autre part de calculateurs situés dans les différentes centrales raccordées au P.H.V. Chacun de ces calculateurs, appelé poste asservi, assure la conduite d'une centrale.

Le P.H.V. permet de mettre à la disposition des Centres inter-régionaux des mouvements d'énergie (C.I.M.E.) les moyens de production d'une vallée. C'est un outil important qui permet aux C.I.M.E. de gérer et de mieux répartir les moyens de production hydraulique sur le réseau électrique national.

A ce titre il exécute les ordres, renseigne le C.I.M.E. et prépare les installations pour permettre :

- en situation normale, l'obtention de bonnes performances du système de production ;
- en situation perturbée, la mise en œuvre rapide des actions élaborées par le C.I.M.E. et destinées à compenser des défaillances ;

- en situation d'incident généralisé, l'exécution des consignes de reconstitution du réseau.

Chaque P.H.V. contrôle les principales centrales hydrauliques d’une zone géographique délimitée.

En ce qui concerne le G.R.P.H. «Alpes", deux P.H.V. sont en cours d'installation : le P.H.V. Oisans et le P.H.V. Maurienne.

Celui de Bourg-d'Oisans contrôle les centrales de Grand'Maison et de Saint-Guillerme, soit $1900 \mathrm{MW}$ de puissance installée.

Celui de Maurienne gère les principales centrales de la vallée de l'Arc: Villarodin, Combe-d'Avrieux, Aussois, Orelle, Bissorte, Super-Bissorte, La Saussaz, Hermillon, ainsi que la centrale du Cheylas, dont le fonctionnement est largement tributaire de l'exploitation de la chaîne de l'Arc.

La réalisation de ces différents projets a nécessité l'installation de calculateurs de deuxième et troisième rang.

Avant de développer les fonctions assurées par ces différents calculateurs, il convient de préciser l'articulation entre les différents niveaux de commande.

Remote control of hydraulic power stations from "postes hydrauliques de vallées » (P.H.V.)

A dozen of hydraulic power stations, some of which are very important nationaly, are or will be remote controled from two POSTES HYDRAULIQUES DE VALLEES (P.H.V.). The purpose of the conference is to look at the step taken to realise the whole computarised command system installed in the P.H.V. and in the power stations. The idea is the creation of a standard software program to direct all operations common to all P.H.V. on one hand, and to all power stations on the other hand. 


\section{Hiérarchie des différents niveaux de commandes}

Pour la conduite automatique des centrales, on distinguera :

\section{Les automates de premier rang}

Ils sont situés à la centrale et affectés à chaque groupe. Ils assurent :

- la mise en sécurité du groupe, lors d'incidents, à partir d'ordres issus des systèmes de protection ;

- l'enchaînement des différentes opérations nécessaires pour le démarrage et l'arrêt du groupe ;

- le réglage des puissances active et réactive du groupe.

\section{Le calculateur de deuxième rang}

Il est normalement situé à la centrale et assure la conduite de l'ensemble des groupes:

- en élaborant ou en recevant de l'extérieur des consignes de conduite globale pour la centrale (ex. : consigne de puissance sur un jeu de barres, consigne de débit...);

- en déterminant les ordres de démarrage/arrêt et les réglages de puissance active ou réactive sur les différents groupes de la centrale.

Pour les centrales raccordées au P.H.V., les calculateurs de second rang sont réalisés à partir de calculateurs de type BULL SPS 5.

\section{Le calculateur de troisième rang}

Il est situé au poste de commande du P.H.V. et constitue l'interface nécessaire entre le Chef de quart du P.H.V. et :

- les calculateurs de deuxième rang;

— les calculateurs du dispatching régional.

Outre ses fonctions naturelles de supervision et de commande, le P.H.V. assure également la coordination automatique de la conduite des centrales hydrauliquement liées.

En période de crue, il permet de renseigner et de coordonner l'action des exploitants présents sur les barrages.

Le système P.H.V. a nécessité d'importants développements logiciels tant pour le poste de commande que pour les postes asservis.

Ces deux projets informatiques ont été conçus et réalisés avec un permanent souci d'homogénéisation des matériels et des logiciels. Cette standardisation a été mise en œuvre avec deux logiques très différentes pour le poste de commande et les postes asservis.

- dans le cas du poste de commande, il est apparu très vite que la majorité des fonctions demandées était identique d'un poste de commande à l'autre. Il a donc été décidé de développer un logiciel d'accueil commun dit logiciel standard, comportant tous les modules d'acquisition, de traitement, de dialogue opérateur et de transmission. Ce logiciel standard prévoit des interfaces simples pour accueillir les fonctions spécifiques à l'exploitation d'un P.H.V. particulier ;

— dans le cas des postes asservis, le G.R.P.H. "Alpes ", après avoir analysé les différents types d'exploitation et les différentes contraintes rencontrées dans nos centrales, a décidé de définir un logiciel unique disposant d'un système d'options qui permet, en partant des caractéristiques de la centrale, de construire le logiciel propre à la centrale concernée.

Nous allons donc maintenant préciser pour chaque niveau hiérarchique, les fonctions associées et la démarche retenue pour aboutir à un logiciel standard.

\section{Les automates de premier rang}

Les fonctions remplies par les automatismes de premier rang n'ont pas été modifiées, seules des adaptations ont été nécessaires pour standardiser l'interface avec les automates de deuxième rang.

\section{Les calculateurs de second rang}

Dans le cadre des P.H.V., le G.R.P.H. "Alpes " a développé un logiciel (PALPES) destiné à être implanté sur les calculateurs de second rang des différentes centrales raccordées aux P.H.V. Oisans, Maurienne et Lyon. Le projet prévoit l'installation de 12 calculateurs.

Le G.R.P.H. «Alpes " s'est fixé quatre objectifs :

1) Diminuer le délai global de réalisation.

2) Diminuer en corollaire, le coût de l'opération globale.

3) Améliorer la fiabilité des systèmes.

4) Rendre la maintenance plus facile et donc plus économique.

Nous définirons donc d'abord les fonctions réalisées par ces calculateurs, puis les caractéristiques des centrales concernées, enfin les principes et la démarche adoptés pour aboutir à un logiciel unique et commun aux 12 postes asservis.

\section{Fonctions assurées par ces calculateurs}

Ces calculateurs de second rang appelés postes asservis (P.A.), sont réalisés à partir de calculateurs de type BULL SPS 5. Chaque P.A. assure la conduite d'un aménagement. Les principales fonctions réalisées sont les suivantes : 
- acquisition des informations en provenance de chaque groupe de la centrale;

- répartition d'une consigne globale de puissance ou de débit sur les différents groupes;

- dialogue avec l'opérateur ;

- gestion des liaisons informatiques avec les calculateurs du poste de commande du P.H.V. et éventuellement avec les automates de premier rang (cas de premier rang réalisés avec des automates programmables).

\section{Caractéristiques des centrales concernées par l'installation d'un poste asservi (P.A.)}

Le G.R.P.H. "Alpes » a pour mission de réaliser 12 P.A. permettant de conduire 16 centrales. En effet, dans le cas du Mont-Cenis, le P.A. pilote un complexe constitué de quatre centrales hydrauliquement très liées, chacun des autres P.A. pilotant une seule centrale. Les caractéristiques et les modes d'exploitation de ces centrales sont très divers.

On trouve en effet :

- Des centrales équipées de groupes hydrauliques très différents :

- centrales de haute chute équipées de turbine Pelton;

- centrales de moyenne chute équipées de turbine Francis ;

- trois stations de transfert d'énergie par pompage (Grand'Maison, Superbissorte, Le Cheylas).

\section{- Des automatismes de premier rang disparates :}

La plupart de ceux-ci sont à relais. La réalisation de ces automatismes s'étant étalée sur une vingtaine d'années, les informations mises à la disposition des calculateurs de second rang sont très hétérogènes.

A noter : la centrale de Saint-Guillerme, réalisée en 1982 est équipée d'automates programmables spécifiques de type $\mathrm{H} 20$.

- Des modes d'exploitation adaptés à chaque aménagement :

- exploitation de centrales disposant de réservoirs importants. Le C.I.M.E. transmet au P.H.V. un programme prévisionnel de production. Ce programme donne demiheure par demi-heure les différentes consignes de production à appliquer ;

- exploitation de stations de transfert d'énergie par pompage. Le C.I.M.E. donne alors des programmes de pompage ou de turbinage ;

- exploitation en action immédiate. Ce type de commande est conçu pour être transmis et déclenché très rapidement sur l'ensemble des centrales téléconduites. Cette commande ne comporte pas de notion de durée et met la centrale considérée dans l'état demandé (ex. : fourniture de " X " mégawatts). Ce type de marche représente $70 \%$ de la conduite des STEP ;

- exploitation d'aménagements hydroélectriques constitués de plusieurs centrales liées hydrauliquement entre elles, par exemple les centrales de : Saussaz, Hermillon. Le système P.H.V. assure dans ces cas la coordination entre les différentes centrales;
- exploitation de centrales au fil de l'eau;

- exploitation du réseau électrique de la centrale : certaines centrales évacuent l'énergie sur un seul jeu de barres, d'autres évacuent sur plusieurs jeux de barres coupables ou non ;

- d'autre part, les caractéristiques propres de chaque aménagement induisent des contraintes spécifiques se traduisant par différentes limitations: de débit, de gradient...

\section{Structure du logiciel}

Le G.R.P.H. "Alpes » a eu le souci de définir une architecture logicielle, matérielle pouvant s'adapter aux contextes et aux différentes contraintes de site rencontrés dans nos aménagements.

Cette standardisation en eu pour effet de dégager :

- une structure matérielle commune;

- une structure logicielle commune;

- un logiciel standard s'intégrant dans ces structures: c'est-à-dire un ensemble de fonctions logicielles et matérielles standard communes à tous les P.A. du G.R.P.H. "Alpes ». Ce logiciel repose sur une architecture modulaire ainsi que sur une base de données bien structurée. Il est composé de plusieurs couches de logiciel.

- Noyau :

Le noyau est le Progiciel MASC 16 développé par BULL. Ce progiciel offre :

- des outils de définition d'une base de données;

- des fonctions standard telles que: l'acquisition des entrées (logiques), des mesures analogiques, et les contrôles afférents, le dialogue opérateur, la mise en suivi de variables, l'historique des événements ;

- une structure d'accueil permettant d'étendre le produit et d'y inclure en les standardisant des fonctions jusqu'à présent redéfinies pour chaque projet. Ces différentes fonctions ont été élaborées par la Subdivision Informatique de conduite du S.P.H.

- Des fonctions communes à tous les P.A. du S.P.H. :

- l'acquisition des mesures numériques et les contrôles afférents,

- l'élaboration d'abaques,

- le dialogue,

- la gestion des liaisons télé-informatiques vers le P.H.V.

et les automates de premier rang,

- la perturbographie,

- la synchronisation horaire,

- la gestion des alarmes.

- Les fonctions communes aux P.A. du G.R.P.H. "Alpes":

La dernière couche de logiciel définie par le G.R.P.H. "Alpes", assure les fonctions de conduite proprement dites. Le travail de standardisation a porté sur trois grands axes :

— définir une base de données commune ; 
- effectuer un découpage modulaire pour les différentes fonctions à assurer ;

- synthétiser les différentes contraintes spécifiques de chaque aménagement afin d'obtenir la même formalisation des contraintes pour tous les aménagements.

Une base de données commune : d'une part, l'interface avec les automatismes de premier rang, qu'il soit à relais ou à base d'automates programmables a été normalisé. Cette normalisation a nécessité d'importants travaux d'adaptation sur les premiers rangs mais était une condition indispensable pour définir cette base de données commune.

D'autre part, les informations échangées avec le poste de commande ont été standardisées en fonction du type d'exploitation retenu sur la centrale.

- marche diagrammée ;

- marche au fil de l'eau ;

- station de transfert d'énergie;

- marche en action immédiate.

Une architecture logicielle modulaire: cette architecture logicielle s'est appuyée sur les travaux du groupe de travail «modules logiciels de conduite d'aménagements hydrauliques ".

L'objectif étant de bien préciser les fonctions à assurer, d'effectuer un découpage en fonctions autonomes les unes par rapport aux autres, de préciser l'enchaînement de ces différentes fonctions. Les fonctions définies sont les suivantes :

- réception et mémorisation des demandes de production ;

- mise en forme des différentes acquisitions faites sur les groupes. Il s'agit de déterminer par exemple l'état du groupe, les groupes raccordés à tel jeu de barres;

- calcul de la consigne de puissance active ou de débit, par jeu de barres, soit à partir d'un algorithme de régulation de niveau, soit à partir des commandes de production (marche diagramme ou action immédiate);

- répartition de cette consigne entre les différents groupes ;

- détermination du nombre de groupes à mettre en fonctionnement ;

- réglage de tension soit en mode " réglage secondaire de tension ", soit en mode " ajustement de tension" ;

- l'asservissement des groupes aux consignes demandées ;

- élaboration d'informations pour le P.H.V.

Un système d'option permet au sein d'un module de préciser des variantes en fonction des caractéristiques de la centrale : nombre de groupes, type d'exploitation retenue.
Synthétiser les contraintes spécifiques: lors de l'étude du Cahier des charges, le concepteur a demandé aux exploitants de remplir pour chaque centrale un questionnaire définissant les caractéristiques principales, les contraintes d'exploitation, les automatismes existants.

Ce questionnaire a notamment fait apparaître un grand nombre de contraintes spécifiques comme: des risques de surdébit, d'entonnement d'air à la cheminée d'équilibre, risque de gel de la conduite forcée. A ces contraintes, correspondaient des consignes d'exploitation exprimées sous des formes très différentes les unes des autres.

Un travail important a été fait pour reformaliser ces contraintes en les exprimant systématiquement :

- soit sous forme de contraintes de débit ;

- soit sous forme de contraintes de gradient (prise ou baisse de charge) ;

- soit sous forme de limitations d'amplitude minimale ou maximale.

Cette synthèse permet d'intégrer ces contraintes dans les fonctions PALPES sous forme d'options.

Les études réalisées avec l'entreprise Cegelec ont permis d'obtenir une réalisation cohérente répondant à nos exigences et aux objectifs précités.

\section{Le calculateur de troisième rang}

Le calculateur de troisième rang est constitué par le Poste de commande (PC) du P.H.V. Ce poste de commande permet la conduite et la surveillance des aménagements hydrauliques d'une ou plusieurs vallées. Il est réalisé à partir de deux calculateurs de type Bull SPS 5 qui fonctionnent en secours mutuel afin d'obtenir une très bonne disponibilité. Le poste de commande assure :

Vis-à-vis des exploitants des aménagements hydrauliques :

- une présence permanente effectuée par un service de quart ;

- la surveillance et la téléconduite des aménagements;

- une meilleure coordination des manœuvres;

- une gestion hydraulique affinée dans les limites fixées par le Service des mouvements d'énergie.

Vis-à-vis du Service des mouvements d'énergie :

- un interlocuteur unique ;

- une qualité de service améliorée en particulier sur le plan de la rapidité d'exécution des manœuvres.

La conception de cette nouvelle génération de poste de commande s'est appuyée sur l'expérience acquise dans ce domaine par le Service de la production hydraulique.

Après avoir réalisé un certain nombre de postes de commande, PCH de Lyon, PCC de Saint-Tulle, Genissiat, Châteauneuf/Rhône, le S.P.H. a décidé de formaliser et de synthétiser l'expérience issue de ces diverses réalisations.

Un document de principe a précisé, d'une part, les performances requises vis-à-vis des Centres des mouvements d'énergie, d'autre part, les principales fonctions nécessaires à un poste de commande. 
Les postes de commande ont des fonctions de surveillance et de conduite hydraulique très différentes d'une vallée à l'autre, liées aux contraintes d'exploitation de la vallée. Cependant nombre de fonctions permettant de réaliser la conduite et la surveillance à distance, sont communes.

Les contraintes de maintenance et d'évolution du parc des systèmes de téléconduite imposent de standardiser matériel et logiciel. Il faut cependant se réserver la possibilité d'intégrer les logiciels spécifiques de chaque vallée.

L'ensemble des systèmes de téléconduite des postes de commande auront donc:

- une structure matérielle commune ;

- une structure logicielle commune;

- un logiciel standard intégrable dans ces structures et correspondant aux fonctions communes.

L'ensemble des éléments standard PC doit permettre d'assurer les fonctions de surveillance et de commande des aménagements de la vallée, sans autre adjonction. Les commandes, consignes ou diagrammes de fonctionnement exécutés sont alors ceux introduits par le Chef de Quart grâce au dialogue opérateur, ou demandés par le Service des mouvements d'énergie depuis le Dispatching via la liaison téléinformatique.

Toutes les fonctions ayant pour objet d'élaborer automatiquement des commandes ou consignes en fonction de critères déterminés font partie des fonctions dites spécifiques au site.

Ce logiciel «standard» traite les informations caractéristiques des aménagements. Celles-ci sont contenues dans une "base de données». On conviendra d'intégrer également dans le logiciel "standard " les outils nécessaires à la création de cette base de données, ainsi que les outils d'adaptation du logiciel standard à une périphérie donnée (type de liaison, synoptique, configuration doublée, etc...).

\section{Fonctions standards}

Ces fonctions sont essentiellement :

- La gestion des liaisons téléinformatiques et du système

- liaisons informatiques vers les postes asservis ;

- liaison vers les calculateurs d'acquisition du système régional de conduite du réseau ;

- liaison vers un calculateur d'acquisition de mesure hydraulique.

- Les gestions des informations

- acquisition des informations ;

- envoi de commandes, consignes;

- traitements et mise en forme de ces informations en vue de leur présentation à l'opérateur.

- Les échanges homme-machine

Le dialogue homme-machine concerne :

- le pupitre opérateur ;

- la console de maintenance ;

- le terminal d'information ;

- la restitution de courbes sur table traçante ;

- la restitution de journaux, bilans, relevés ;

- l'affichage d'images et de courbes sur écran.

- Fonctions spécifiques

Le logiciel standard permet d'intégrer les fonctions nécessaires à l'exploitation particulière d'une vallée. On peut citer à titre d'exemple :

- le contrôle de la faisabilité des programmes demandés ;

- la programmation d'éclusées ;

- le programme d'optimisation partielle ou totale de la vallée ;

- l'aide à la conduite en période perturbée (renvoi de tension, tenue en fréquence du réseau).

\section{Conclusion}

La réalisation des Postes de Commande Oisans et

Maurienne par Sodeteg-TAI a montré que $90 \%$ du logiciel d'application est constitué par le noyau standard, $10 \%$ par les fonctions spécifiques. Ces chiffres justifient pleinement la démarche entreprise pour standardiser.

Actuellement le poste de commande de Bourg d'Oisans est en cours de validation. En ce qui concerne le P.H.V. Maurienne, les centrales de Superbissorte et du MontCenis sont actuellement pilotées depuis le poste de commande. Le raccordement des autres P.A. conçus par le G.R.P.H. "Alpes " s'effectuera au second semestre 91. 

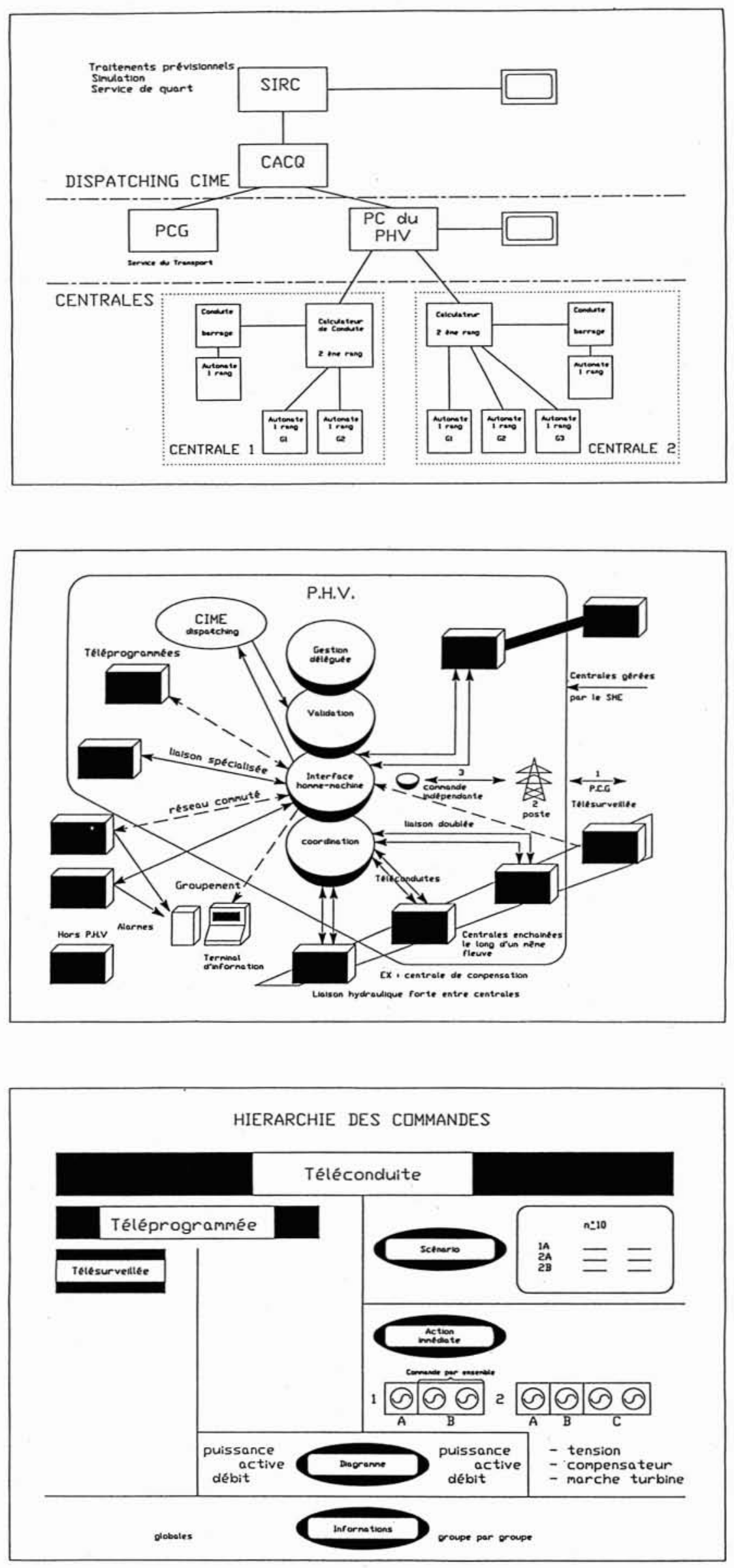

LA HOUILLE BLANCHE/N`2-1991 